\title{
NdRhSn: A ferromagnet with an antiferromagnetic precursor
}

\author{
M. Mihalik,,${ }^{1,2,}$ J. Prokleška, ${ }^{1,3}$ J. Kamarád, ${ }^{4}$ K. Prokeš, ${ }^{5}$ O. Isnard, ${ }^{3}$ G. J. McIntyre, ${ }^{3}$ A. Dönni, ${ }^{2}$ S. Yoshii, ${ }^{6}$ \\ H. Kitazawa, ${ }^{2}$ V. Sechovský, ${ }^{1}$ and F. R. de Boer ${ }^{1,6,7}$ \\ ${ }^{1}$ Charles University, Faculty of Mathematics and Physics, Department of Condensed Matter Physics, Ke Karlovu 5 , \\ CZ-121 16 Prague 2, Czech Republic \\ ${ }^{2}$ National Institute for Materials Science, 1-2-1 Sengen, Tsukuba, Ibaraki 305-0047, Japan \\ ${ }^{3}$ Institut Laue Langevin, BP 156, 38042, Grenoble, France \\ ${ }^{4}$ Institute of Physics of the AS CR, Cukrovarnická 10/112, CZ-162 00, Prague 6, Czech Republic \\ ${ }^{5}$ Helmholtz Zentrum Berlin, Hahn-Meitner Platz, 1, D-14 109, Berlin, Germany \\ ${ }^{6}$ KYOKUGEN, Osaka University, Toyonaka, Osaka 560-8531, Japan \\ ${ }^{7}$ Van der Waals-Zeeman Instituut, Universiteit van Amsterdam, Amsterdam, The Netherlands \\ (Received 11 October 2010; revised manuscript received 17 December 2010; published 9 March 2011)
}

\begin{abstract}
$\mathrm{NdRhSn}$ has a ferromagnetic ground state $\left[T_{t}=7.6(1) \mathrm{K}\right]$ with a magnetic moment equal to $2 / 3$ of the free $\mathrm{Nd}^{3+}$-ion moment. Upon cooling from the paramagnetic state, the ferromagnetism is preceded by an incommensurate antiferromagnetic state between $T_{N}=9.8(1) \mathrm{K}$ and $T_{t}$. In both magnetic phases the magnetic moments are locked along the $c$ axis, which is the easy-magnetization axis of the system. We have investigated this unusual situation by measuring anomalies of several bulk physical properties characteristic for the magnetic phase transitions at $T_{N}$ and $T_{t}$ and the response of these properties to an applied magnetic field. Neutron-diffraction experiments have been also performed at low temperatures. Furthermore, in order to understand better the physical properties of NdRhSn, single crystals have been studied under external hydrostatic and uniaxial pressure and in high magnetic fields. An antiferromagnetic phase with a propagation vector $\left(\begin{array}{ll}0 & 0 \\ 1 / 1 & 11\end{array}\right)$ has been found between $T_{N}$ and $T_{t}$. The magnetic-ordering temperatures $T_{N}$ and $T_{t}$ are sensitive to both hydrostatic and uniaxial pressures, but in a different manner. Application of hydrostatic pressure leads to a reduction of the ordering temperatures at rates $\Delta T_{N} / \Delta p=-0.76(5) \mathrm{K} / \mathrm{GPa}$ and $\Delta T_{t} / \Delta p=-0.9(2) \mathrm{K} / \mathrm{GPa}$ while uniaxial pressure applied along the $c$ axis leads to an increase of the ordering temperatures at rates $\Delta T_{N} / \Delta p=+1.2(5) \mathrm{K} / \mathrm{GPa}$ and $\Delta T_{t} / \Delta p=$ +2.7 (4) $\mathrm{K} / \mathrm{GPa}$. No considerable influence of pressure on the antiferromagnetic propagation vector has been found. The peculiar evolution of magnetism in $\mathrm{NdRhSn}$ with temperature indicates a complex hierarchy of exchange interactions.
\end{abstract}

DOI: 10.1103/PhysRevB.83.104403 PACS number(s): 75.30.-m, 65.40.-b, 72.15.Eb, 75.50.Ee

\section{INTRODUCTION}

$R E T X$ compounds $(R E=$ rare earth; $T=d$ metal; $X=$ $p$ metal) crystallize in a variety of structure types, depending mainly upon the nature of the $T$ and $X$ elements. ${ }^{1}$ The $R E T X$ compounds with fixed $T$ and $X$ elements are usually isostructural, which allows the study of magnetism and related properties for a series of $R E$ elements in the same crystallographic environment.

$R E \mathrm{RhSn}$ compounds crystallize in the hexagonal $\mathrm{ZrNiAl}$ type of structure, which is a ternary-ordered variant of the binary $\mathrm{Fe}_{2} \mathrm{P}$ type of structure. ${ }^{2}$ Among the $R E \mathrm{RhSn}$ compounds with light $R E \mathrm{~s}$, LaRhSn has been found superconducting below $2 \mathrm{~K},{ }^{3,4} \mathrm{CeRhSn}$ has been reported to be a valence fluctuator, ${ }^{5}$ and ferromagnetism has been reported for PrRhSn below $3 \mathrm{~K}^{6,7}$

Concerning the magnetism in NdRhSn, only scarce information on the behavior of polycrystalline samples has been published thus far. Routsi et al. ${ }^{8}$ have found that, above $40 \mathrm{~K}$, the magnetic susceptibility obeys a Curie-Weiss law with an effective moment $\mu_{\mathrm{eff}}=3.55 \mu_{B} /$ f.u. and a paramagnetic Curie temperature $\Theta_{p}=12 \mathrm{~K}$. Ea̧tka et al. ${ }^{9}$ have reported a paramagnetic-ferromagnetic phase transition at $T_{C}=10.3 \mathrm{~K}$ and a superconducting transition at $T_{\mathrm{SC}}=6.9 \mathrm{~K}$. Later, Łątka et $a l .{ }^{10}$ have reported only the ferromagnetic phase transition to occur in this compound. They have also measured ${ }^{119} \mathrm{Sn}$ Mössbauer spectra at various temperatures and have also determined the temperature dependence of the hyperfine-field distribution function, $F\left(H_{\mathrm{hf}}\right)$, which is rather narrow below $7 \mathrm{~K}$ and very broad in the temperature range $7<T<10 \mathrm{~K}$, which is not compatible with ferromagnetism existing in this temperature range. This confusing situation has stimulated us to grow single crystals of this compound and to investigate its physical properties more thoroughly. Recently, we have published magnetization, ac-susceptibility, and specific-heat results, ${ }^{11,12}$ which clearly indicate that $\mathrm{NdRhSn}$ orders antiferromagnetically at $T_{N}=9.8 \mathrm{~K}$ and undergoes an order-to-order magnetic phase transition at $T_{C}=7.6 \mathrm{~K}$ to the ferromagnetic ground state with a spontaneous moment corresponding to $\sim 2 / 3$ of the free $\mathrm{Nd}^{3+}$-ion moment. Owing to arguments presented in this paper, it has appeared that the use of " $T_{C}$ " for the transition at $7.6 \mathrm{~K}$ is slightly misleading, because it is not the paramagnetic-ferromagnetic transition. From now on, we change the notation to $T_{t}$. The ferromagnetism with an antiferromagnetic precursor is rather uncommon in materials that conserve the crystal structure in the involved temperature region. To our knowledge, only $\mathrm{PrRu}_{2} \mathrm{Si}_{2},{ }^{13} \mathrm{Co}_{3} \mathrm{~V}_{2} \mathrm{O}_{8},{ }^{14}$ and Dy (Ref. 15) are analogous cases.

In this paper, we extend our study of $\mathrm{NdRhSn}$ with additional macroscopic measurements (specific heat, thermal expansion, magnetostriction, electrical resistivity, magnetization under applied hydrostatic, and uniaxial pressure and magnetization in high magnetic fields) and by microscopic measurements (powder and single-crystal neutron diffraction). 
TABLE I. Comparison of crystallographic data of $\mathrm{NdRhSn}$ at different temperatures and obtained by different experimental techniques. *Powder prepared from a polycrystalline sample; **powder prepared from a single crystal. R.T. means room temperature.

\begin{tabular}{lcllc}
\hline \hline Experiment & $T(\mathrm{~K})$ & $a(\mathrm{~nm})$ & $c(\mathrm{~nm})$ & Reference \\
\hline \multirow{3}{*}{ Powder XRD } & R.T. & $0.7413(1)$ & $0.41290(9)$ & $10^{*}$ \\
& R.T. & $0.7399(3)$ & $0.4127(3)$ & $11^{* *}$ \\
& R.T. & $0.7399(2)$ & $0.4128(2)$ & This work* \\
Powder neutron & R.T. & $0.742(1)$ & $0.418(3)$ & This work* \\
diffraction & 14 & $0.741(1)$ & $0.414(1)$ & This work* \\
& 2 & $0.741(1)$ & $0.415(1)$ & This work* \\
\hline \hline
\end{tabular}

\section{SAMPLE PREPARATION AND CHARACTERIZATION}

Polycrystalline NdRhSn was prepared by arc melting under a protective Ar atmosphere. Two single crystals were prepared by the Czochralski method: The first one, prepared in the tri-arc furnace at the Department of Condensed Matter Physics (DCMP), Charles University, Prague, was used for the bulk measurements presented in previous papers ${ }^{11,12}$ and for the resistivity, high-field magnetization, and thermal-expansion measurements in the present paper. The second single crystal was prepared in a tetra-arc furnace at the National Institute for Materials Science (NIMS), Tsukuba, and was used for neutron-diffraction experiments and magnetization measurements under hydrostatic and uniaxial pressure. In all cases, stoichiometric amounts of $\mathrm{Nd}, \mathrm{Rh}$, and $\mathrm{Sn}$ of at least $3 \mathrm{~N}$ nominal purity were used. For the preparation of both crystals, we have used either a tungsten rod or a tungsten wire as a seed, applied a random-necking procedure, and used pulling speeds between 6 and $10 \mathrm{~mm} / \mathrm{h}$. The grown single crystals were 20-25 mm long with a maximum diameter $\sim 4 \mathrm{~mm}$.

The polycrystalline sample and parts of both single crystals were checked by $\mathrm{x}$-ray powder diffraction (XRD) to ensure that they are single phase with the proper $\mathrm{ZrNiAl}$ crystal structure. The lattice parameters, determined from the diffraction data by Rietveld refinement incorporated in the FULLPROF software, ${ }^{16}$ are tabulated in Table I. The crystals were additionally investigated by Laue diffraction to ensure that they are single crystalline.

\section{EXPERIMENTAL}

Powder neutron-diffraction experiments were performed on the D1B diffractometer at the Institut Laue Langevin (ILL), Grenoble, using the standard ILL orange cryostat. We have collected diffraction patterns at 2, 4.3, 9.6, and $14 \mathrm{~K}$ with higher statistics and several diffraction patterns in the 4.3-20 K temperature range with lower statistics to follow the temperature evolution of the intensities of the reflections. In all scans, we have used a monochromatic neutron beam with a wavelength $\lambda=2.52 \AA$ and collected data at scattering angles in the $3^{\circ}<2 \theta<82^{\circ}$ range.

Single-crystal neutron diffraction at ambient pressure was also performed at ILL, using the VIVALDI diffractometer with the standard orange cryostat. Laue patterns were taken at several orientations and selected temperatures. Special care was given to measurements in the temperature interval where the antiferromagnetic phase was expected.
Neutron-diffraction experiments under applied hydrostatic pressure and uniaxial stress were performed on the E4 diffractometer, equipped with a two-dimensional detector in combination with the $4 \mathrm{~T}$ horizontal magnet HM2 in the Helmholtz Zentrum, Berlin. In this experiment, we have used monochromatic neutrons with a wavelength $\lambda=2.461 \AA$. The magnetic field had to be applied $56^{\circ}$ from the $c$ axis in order to allow for the desired reciprocal space range of the incoming and diffracted neutrons to pass through the windows of the magnet. Considering the very strong uniaxial magnetic anisotropy in $\mathrm{NdRhSn},{ }^{11}$ the projection of the magnetic field into the direction of the $a$ axis has no significant effect on the magnetism of $\mathrm{NdRhSn}$ and that only the projection of the magnetic field on the $c$-axis direction affects the magnetic state. All presented magnetic fields in this neutron-diffraction experiment are the calculated projections of the applied magnetic field on the $c$-axis. The hydrostatic pressure was produced by a $\mathrm{CuBe}$ clamped-piston cell with manganin wire as a pressure sensor. For the uniaxial pressure, which was applied along the $c$ axis, the pressure cell described in Ref. 17 was used. In both pressure experiments, we have studied the (2 $\left.\begin{array}{lll}2 & 0\end{array}\right)$ reflection and its magnetic satellites. Data were collected in the temperature range $2-15 \mathrm{~K}$.

Measurements of the thermal expansion and magnetostriction along the two main crystallographic axes were performed at temperature range $2.5-20 \mathrm{~K}$ using a capacitance cell ${ }^{18}$ and a physical property measurement system (PPMS) from Quantum Design. Because the $c$ axis is in the easy-magnetization direction (EMD) of NdRhSn, ${ }^{11}$ we have measured the magnetostriction only in magnetic fields applied along this axis. The thermal-expansion data were taken during heating with a speed of $0.05-0.10 \mathrm{~K} \mathrm{~min}^{-1}$. Cooling of the samples was always performed in the zero magnetic field.

Magnetization measurements in pulsed fields up to $50 \mathrm{~T}$ were carried out at $4.2 \mathrm{~K}$ at the High Magnetic Field Laboratory, KYOKUGEN, Osaka University, Japan, in a nondestructive pulse magnet. Two kinds of measurements have been performed on a single crystal. The saturation magnetization was measured on a crystal that was not fixed in the sample holder so that it could be rotated freely by the applied field with its $c$ axis (EMD) into the field direction. Measurement of the magnetization in the hard-magnetization direction (HMD) was done with the $c$ axis fixed perpendicular to the external field. Unfortunately, a misorientation of the sample of the order of $8^{\circ}$ has caused the presence of a small component of the $c$-axis magnetization that has been corrected using the data obtained from the first experiment.

The temperature and field variations of the magnetization under external pressure were measured by using two different clamped-piston hydrostatic-pressure cells and a uniaxial pressure cell. All three cells are designed to operate inside the magnetic property measurement system (MPMS) (Quantum Design) superconducting qunatum interference device (SQUID) magnetometer. The first hydrostatic experiment (experiment No. 1) was done at NIMS, Tsukuba. In this experiment, the internal pressure was deduced from the applied external force during the clamping of the cell. The second hydrostatic pressure experiment (experiment No. 2) was done at the Charles University in Prague using the cell described in Ref. 19. The pressure was determined using the pressure 
dependence of the superconducting-transition temperature of $\mathrm{Pb},{ }^{20}$ which was enclosed as a sensor. The uniaxial-pressure experiments were carried out at the Institute of Physics of the Academy of Sciences of the Czech Republic (IOP AS, CR), Prague, using the uniaxial-pressure cell described in Ref. 17. In this case, the yield stress was produced by Bellville springs and the pressure was determined by dividing the force produced by squeezed Bellville springs by the sample cross section.

Using the options implemented in the mentioned PPMS apparatus, we have performed measurements of the specific heat, electrical resistivity, and magnetoresistance of the single crystal and of polycrystalline $\mathrm{NdRhSn}$ samples in the temperature range from $2 \mathrm{~K}$ up to $20 \mathrm{~K}$. As for the resistivity measurements, owing to the small dimensions of the samples, only the relative values of the electrical resistivity and transverse magnetoresistivity are presented.

\section{RESULTS AND DISCUSSIOIN}

\section{A. Specific heat}

The specific heat of the single-crystal and polycrystalline $\mathrm{NdRhSn}$ samples is shown in Fig. 1(a). For both samples we see two anomalies: the lower-temperature sharp lambda-type anomaly, which occurs at temperature $T_{t}$, and a broader, nonsymmetric peak at temperature $T_{N}$. For the single crystal, $T_{t}=7.6(1) \mathrm{K}$ and $T_{N}=9.8(1) \mathrm{K}$. For the polycrystalline sample, the peak corresponding to $T_{N}$ occurs also at $9.8(1) \mathrm{K}$, but the lower transition is slightly shifted to $T_{t}=7.9(1) \mathrm{K}$. These results suggest that $T_{t}$ is slightly sample dependent. Nevertheless, $T_{t}$ is observable for both samples of different quality.

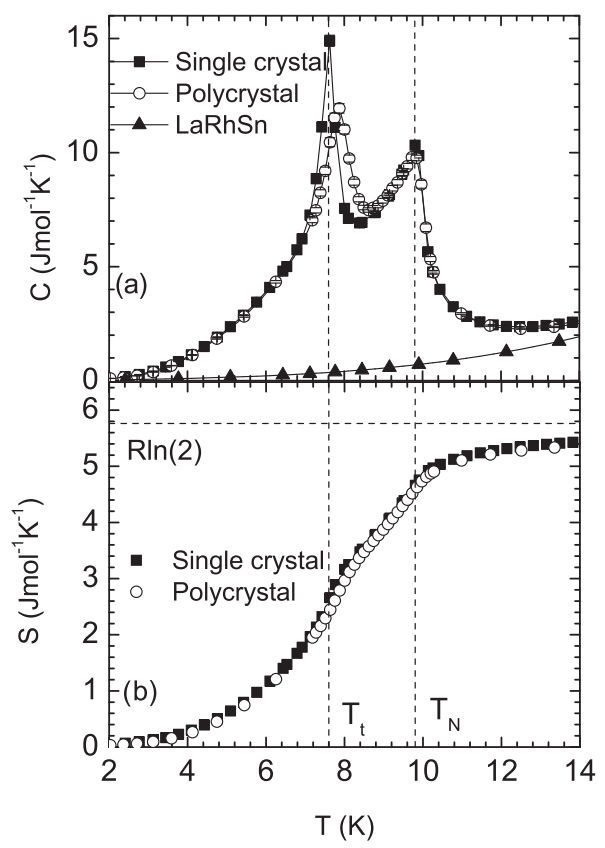

FIG. 1. (a) Temperature dependence of the specific heat of $\mathrm{NdRhSn}$ measured on a single crystal and polycrystal compared with the specific heat of LaRhSn. The NdRhSn single-crystal data are taken from Mihalik et al. (Ref. 11) and the LaRhSn data are taken from Mihalik et al. (Ref. 7). (b) The calculated magnetic entropy where LaRhSn has been used as a nonmagnetic analog.
To calculate the magnetic entropy of $\mathrm{NdRhSn}$ in the temperature region $2<T<14 \mathrm{~K}$, LaRhSn has been used as a nonmagnetic analog. ${ }^{7}$ Below $2 \mathrm{~K}$, the magnetic entropy was estimated using linear extrapolation to $S=0 \mathrm{~J} \mathrm{~mol}^{-1} \mathrm{~K}^{-1}$ at $T=0 \mathrm{~K}$. The resulting magnetic entropy [Fig. 1(b)] exhibits practically no difference between the polycrystalline sample and the single crystal. The magnetic entropy reaches values of $2.44 \mathrm{~J} \mathrm{~mol}^{-1} \mathrm{~K}^{-1}$ at $T_{t}, 4.63 \mathrm{~J} \mathrm{~mol}^{-1} \mathrm{~K}^{-1}$ at $T_{N}$, and saturates to a value of $5.4 \mathrm{~J} \mathrm{~mol}^{-1} \mathrm{~K}^{-1}$ at higher temperatures. The last value of the magnetic entropy is still somehow lower than $R \ln (2)\left(=5.76 \mathrm{~J} \mathrm{~mol}^{-1} \mathrm{~K}^{-1}\right)$. To find the magnetic entropy connected with $T_{t}$ and $T_{N}$, we need to separate both peaks in the specific heat from each other. Because these two peaks have a significant overlap and at least the peak connected with $T_{N}$ is highly asymmetric, we decided to separate the peaks ad hoc in the minimum in the specific heat $(=8.4 \mathrm{~K})$. After this separation we have estimated the entropy connected with $T_{t}$ to be $3.4 \mathrm{~J} \mathrm{~mol}^{-1} \mathrm{~K}^{-1}$ and entropy connected with $T_{N}$ to be $2 \mathrm{~J} \mathrm{~mol}^{-1} \mathrm{~K}^{-1}$.

\section{B. Electrical resistivity and magnetoresistivity}

The electrical resistivity of the compound monotonously decreases by decreasing temperature, and shows the metallic behavior at temperatures higher than $20 \mathrm{~K}$ and two anomalies connected with $T_{t}$ and $T_{N}$ at lower temperatures (see Fig. 2). The observed residual resistivity ratio (defined as $\rho_{300 \mathrm{~K}} / \rho_{2} \mathrm{~K}$ ) is 9.3 for the $a$ axis and 10.1 for the $c$ axis. The singlecrystal resistivity exhibits similar features as the measurements on a polycrystal. ${ }^{9}$ The two anomalies occur at the same temperatures as in the specific heat (see Sec. IV A) and magnetization (see Mihalik et al. ${ }^{11}$ ). The resistivity remains unchanged if a magnetic field is applied along the $a$ axis. On the other hand, application of the magnetic field along the

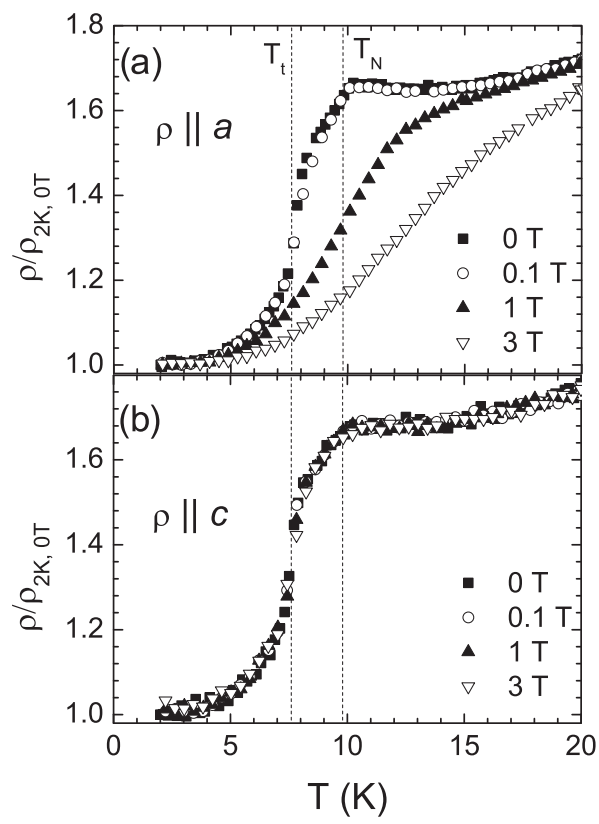

FIG. 2. Relative electrical resistivity and magnetoresistivity of NdRhSn for (a) $\rho \| a$ axis, $B \| c$ axis and (b) $\rho \| c$ axis, $B \| a$ axis. The dashed lines represent the positions of $T_{N}$ and $T_{t}$. All data were obtained during the increasing of the temperature. 
$c$ axis has a dramatic effect on the electrical resistivity: the change from the two-anomaly curve at a magnetic field of $0.1 \mathrm{~T}$ into a curve with only one anomaly at a magnetic field of $1 \mathrm{~T}$ [Fig. 2(a)]. This change is related to the field-induced changes of the magnetic structure observed previously by the magnetization and specific-heat measurements. ${ }^{11}$

It should be mentioned that no superconductivity exists in NdRhSn above $2 \mathrm{~K}$, which is in contradiction with the finding of Łatka et al. ${ }^{9}$ On the other hand, the resistivity presented in Fig. 1 of Łătka's paper ${ }^{9}$ does not drop to zero at any temperature. There exist two other explanations for the kink at $6.9 \mathrm{~K}$ in Łątka's paper: ${ }^{9}$ Either there is some impurity presented in the sample, or the kink is associated with $T_{t}$. The supportive argument for the first option is that also the CeRhSn sample used in this paper was not pure [compare the data published in Ref. 9 with other papers dealing with CeRhSn (Refs. 5 and 21)]. The supportive argument for the second scenario is that $6.9 \mathrm{~K}$ is close to $T_{t}$ and on top of it, in Fig. 3(b) of Łątka et al., ${ }^{9}$ the authors present a highly nonsymmetric minor hysteresis loop that light a suspicion that the zero of their magnetometer was shifted. Then the diamagnetic signal presented in Fig. 1(b) in Łątka et al. ${ }^{9}$ can be just an coincidence with this zero shift.

\section{High-field experiment}

The saturated magnetic moment of NdRhSn along the $c$ axis reported in previous work ${ }^{11}$ reaches only $2 / 3$ of the $\mathrm{Nd}^{3+}$ freeion moment. This implies two possible scenarios: Either the $\mathrm{Nd}$ ions possess the full moment of the free $\mathrm{Nd}^{3+}$ ion and the magnetic field of $14 \mathrm{~T}$ is not large enough to orient all moments into the direction of the applied field, or the $\mathrm{Nd}$ ions do not have the moment of a free $\mathrm{Nd}^{3+}$ ion. To investigate the possibility of an additional magnetic phase transition, magnetization measurements in magnetic fields up to $50 \mathrm{~T}$ have been performed.

Except for the previously published metamagnetic phase transition in the antiferromagnetic phase in a field of $\sim 0.1 \mathrm{~T},{ }^{12}$ the high-field experiment (Fig. 3) does not reveal an additional phase transition at higher fields. The magnetization measured on the single crystal, which could freely rotate in the applied field and thence orient the $c$ axis (easy-magnetization axis) into the direction of the magnetic field, reaches only $2.5 \mu_{B}$ at $50 \mathrm{~T}$. The increase of the magnetization above $20 \mathrm{~T}$ may be ascribed to a paramagnetic contribution. In the case of magnetic

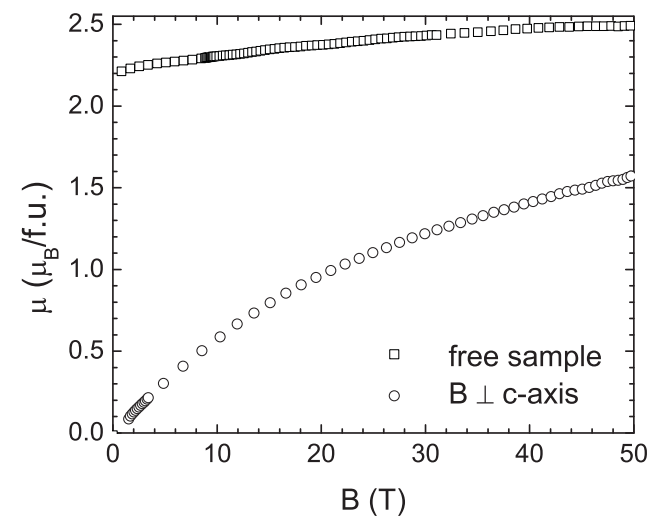

FIG. 3. Magnetization at $T=4.2 \mathrm{~K}$ in fields up to $50 \mathrm{~T}$. field applied perpendicular to the $c$ axis, we have found the monotonous increase of the magnetization reaching only $1.55 \mu_{B} /$ f.u. at $50 \mathrm{~T}$. If we can afford the linear extrapolation, we estimate that the magnetization will reach $2.5 \mu_{B} /$ f.u. at a magnetic field of $100 \mathrm{~T}$ applied perpendicular to the $c$ axis. It points to the large magnetocrystalline anisotropy in NdRhSn.

Based on the high-field experiment, the scenario that the $\mathrm{Nd}$ ions do not possess the full magnetic moment of the free $\mathrm{Nd}^{3+}$ ion seems to be the correct one. The reduction of the neodymium magnetic moment and the large magnetocrystalline anisotropy are the consequences of the strong crystal field in NdRhSn.

\section{Neutron diffraction at ambient pressure}

All powder neutron-diffraction patterns measured at temperatures above $10 \mathrm{~K}$ could be fitted using the crystallographic structure as determined by XRD. However, below $10 \mathrm{~K}$, additional magnetic reflections appear (Fig. 4). To estimate the magnetic contribution to the powder pattern, we have normalized all diffraction patterns to the same monitor and then subtracted the $14 \mathrm{~K}$ pattern from all patterns. These difference patterns display an increase of the intensity of some nuclear peaks for the pattern collected at $4.3 \mathrm{~K}$, which suggests that the ground-state magnetic structure of $\mathrm{NdRhSn}$ is commensurate with the crystal structure. The intensity of the $\left(\begin{array}{lll}0 & 0 & 1\end{array}\right)$ and (llll $\left.\begin{array}{lll}0 & 2\end{array}\right)$ reflections does not increase when the temperature is lowered, which is in accordance with the magnetization results

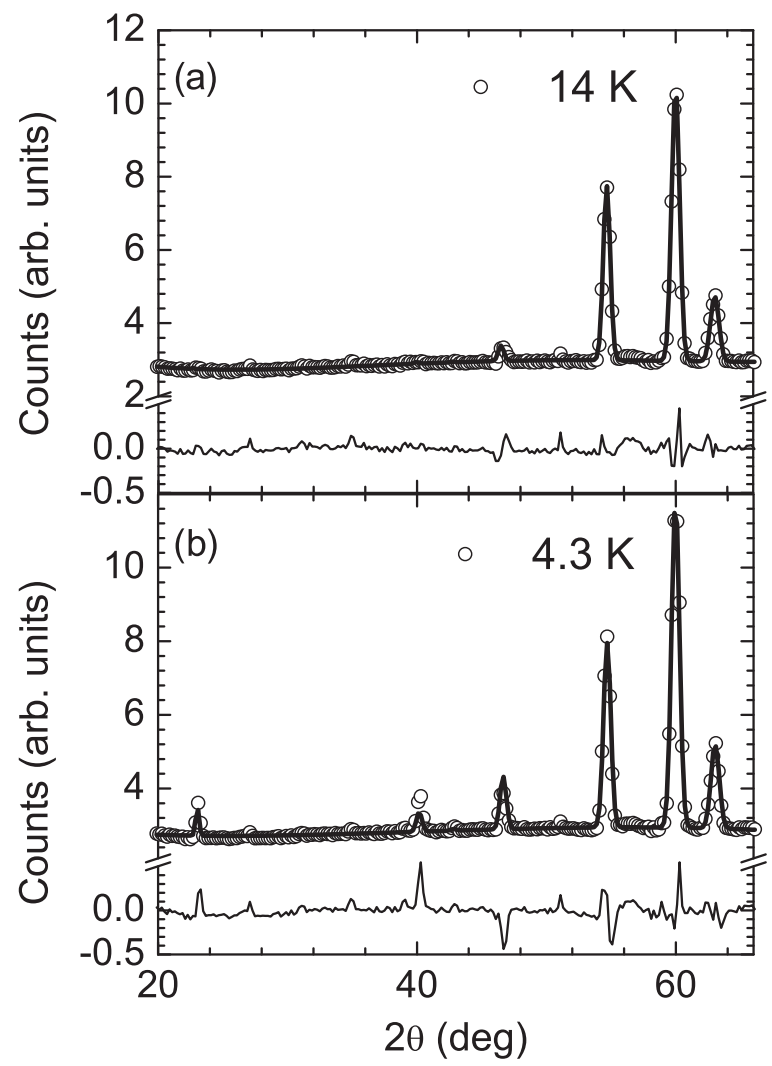

FIG. 4. Representative powder neutron-diffraction patterns of NdRhSn at (a) $14 \mathrm{~K}$ and (b) $4.3 \mathrm{~K}$. The circles represents the experimental data, the line through circles represents the best Rietveld fit, and the line below the circles represents the difference. 
that indicate that the magnetic moments are aligned along the $c$ axis. The magnetic part of the diffraction patterns has been fitted in the whole $3^{\circ}<2 \theta<82^{\circ}$ range using the program FULLPROF $^{16}$ based on the Rietveld method. Because in many RETX compounds [for example, PrPdSn and NdPdSn, ${ }^{22}$ $R$ PdIn ( $R=\mathrm{Nd}$, Ho, Er), ${ }^{23} \mathrm{PrPdAl},{ }^{24}$ and many others] only a magnetic moment on the $R$ ions was observed, we have used a model of a pure ferromagnet with magnetic moments only on the $\mathrm{Nd}^{3+}$ ions and aligned along the $c$ axis as a starting model. The fit converged perfectly for all patterns collected below $7.5 \mathrm{~K}$. The final Rietveld $R$ factors were $R_{p}=11.3$, $R_{\mathrm{wp}}=9.25, \chi^{2}=10.3$, and $R_{B}=1.76$ for the $14 \mathrm{~K}$ data and $R_{p}=14.7, R_{\mathrm{wp}}=12.1, \chi^{2}=44.4$, and $R_{B}=3.97$ for the $4.3 \mathrm{~K}$ data. The match between the experimental data and the best fit is visualized in Fig. 4. We have made also a full symmetry analysis to derive all possible magnetic structures in order to check if there is a magnetic structure with a $c$-axis ferromagnetic component that describes better the observed data. However, it appeared that this is not the case.

The obtained $\mathrm{Nd}$ moment is $2.4(2) \mu_{B}$ at $4.3 \mathrm{~K}$ and decreases to a value of $1.5(2) \mu_{B}$ at $7.2 \mathrm{~K}$. The former value is in very good agreement with the saturation-magnetization moment of $2.24 \mu_{B}$ /f.u. obtained in bulk magnetization measurements reported earlier, ${ }^{11}$ while it is somewhat lower than the value of $2.5 \mu_{B}$ measured at $50 \mathrm{~T}$. The magnetic moment is substantially lower than the magnetic moment of the free $\mathrm{Nd}^{3+}$ ion $\left(3.27 \mu_{B}\right)$. Low values of the $\mathrm{Nd}$ magnetic moment from $1.7 \mu_{B}$ up to $2.5 \mu_{B}$ have been observed in most Nd-based intermetallic compounds, ${ }^{25}$ including the $\mathrm{Nd} T X$ compounds [for example, $\mu=1.94(4) \mu_{B}$ in NdPdSn, ${ }^{22} \mu=1.8(1) \mu_{B}$ in NdPdIn, ${ }^{23}$ and $\mu=2.42(9) \mu_{B}$ in NdNiSn (Ref. 26)] and it seems that the $\mathrm{NdRhSn}$ is not an exception.

In the patterns collected above $7.5 \mathrm{~K}$, no additional reflections have been found as compared to the patterns below $7.5 \mathrm{~K}$. However, the ratio of the intensities of the observed reflections changes drastically, indicating that the magnetic phase at temperatures above $7.5 \mathrm{~K}$ is not the same as below $7.5 \mathrm{~K}$. The statistics of the neutron-diffraction experiment is poor for temperatures above $7.5 \mathrm{~K}$ and, therefore, this experiment does not enable to specify the magnetic phase between $T_{t}$ and $T_{N}$.

To investigate the magnetic structure between $T_{t}$ and $T_{N}$, a single-crystal neutron-diffraction experiment has been carried out that reveals additional reflections of magnetic origin below $10 \mathrm{~K}$ with noninteger $h k l$ indices (Fig. 5). The intensity of these reflections increases with decreasing temperature, has a maximum at $\sim 8 \mathrm{~K}$, and vanishes below $7 \mathrm{~K}$. In total, 477 independent reflections of magnetic origin with noninteger $h k l$ indices have been observed. All of them can be indexed in agreement with the propagation vector $(00$ 1/11) [Fig. 5(b)], indicating the existence of a sine-modulated or squared-up antiferromagnetic structure in the temperature range between $T_{t}$ and $T_{N}$. We have to note that the estimated intensities of the higher harmonics based on the observed intensity of the magnetic reflections suggested that there is no chance to spot the higher harmonics on the VIVALDI instrument. That is why the question if the structure is a sine-modulated or a squared-up antiferromagnetic remains open at this stage in the research.

Inspection of the Laue patterns taken between $T_{t}$ and $T_{N}$ shows that the obtained propagation vector is temperature inde-

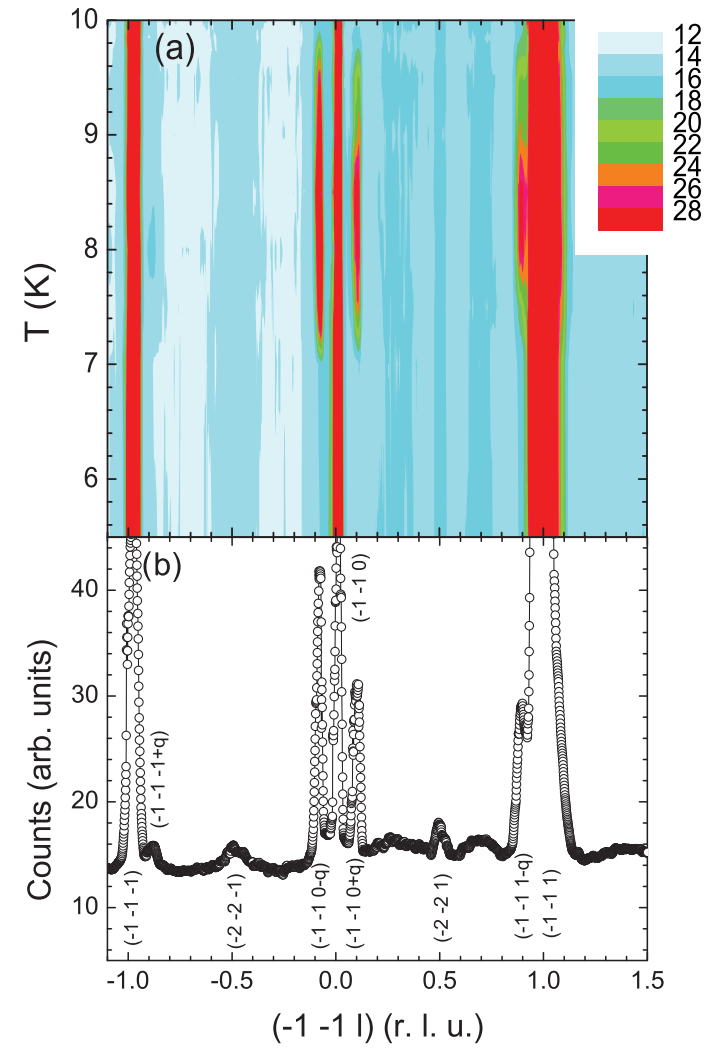

FIG. 5. (Color online) Cut from the neutron Laue pattern of NdRhSn: (a) Temperature evolution; (b) indexed cut at $8.5 \mathrm{~K}$.

pendent within the experimental error. The magnetic moments in this phase are aligned along the $c$ axis. Below $T_{t}$, however, some nuclear reflections gain in intensity (Fig. 6), which is in good agreement with the powder neutron-diffraction results. It indicates that, below $T_{t}$, the crystallographic and the magnetic unit cell are commensurate. The previously performed bulk-magnetization measurements ${ }^{11}$ and the neutron-diffraction results allow us to conclude that the ground state of NdRhSn is indeed ferromagnetic with a $\mathrm{Nd}$ moment

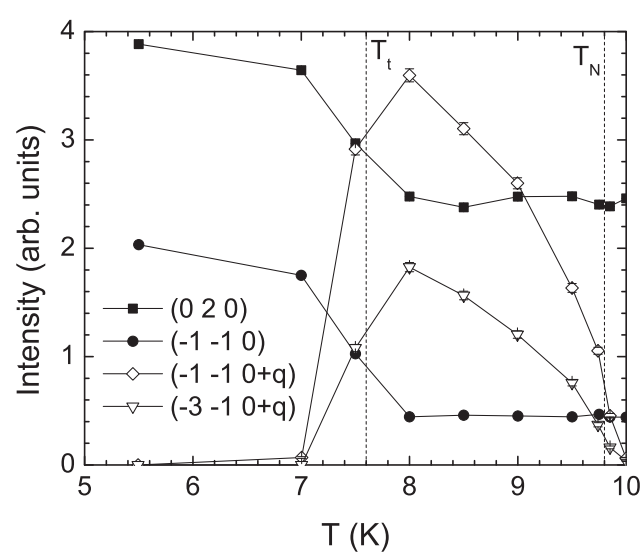

FIG. 6. Temperature evolution of the integrated intensities of representative reflections. The intensity of the magnetic satellites has been multiplied by a factor of 1000 to match the scale of the plot. The dashed lines indicate $T_{N}$ and $T_{t}$. 
that is considerably smaller than the free $\mathrm{Nd}^{3+}$-ion value. Also in this case, the magnetic moments are aligned along the $c$ axis.

The ${ }^{119} \mathrm{Sn}$ Mössbauer spectroscopy experiment of Łatka et $a l .{ }^{10}$ showed a very narrow field-distribution function of the hyperfine field, acting on the $\mathrm{Sn}$ ion below $7 \mathrm{~K}$. However, this distribution function is several times broader for the temperature region $8<T<9.25 \mathrm{~K}$ (Fig. 11 in Ref. 10). In our opinion, this broadening is directly connected with the change of the magnetic structure from the simple ferromagnetic to an other, probably long-range modulated, structure: In the ferromagnetic state all Sn nuclei experience the uniform hyperfine field, but if the structure is long-range modulated, the $\mathrm{Sn}$ atoms experience different hyperfine fields, depending on their position in the magnetic unit cell.

It is worth mentioning that, within the experimental error in the polycrystalline and single-crystal neutron-diffraction experiment, no change of the crystal structure was found in the temperature range $2-300 \mathrm{~K}$.

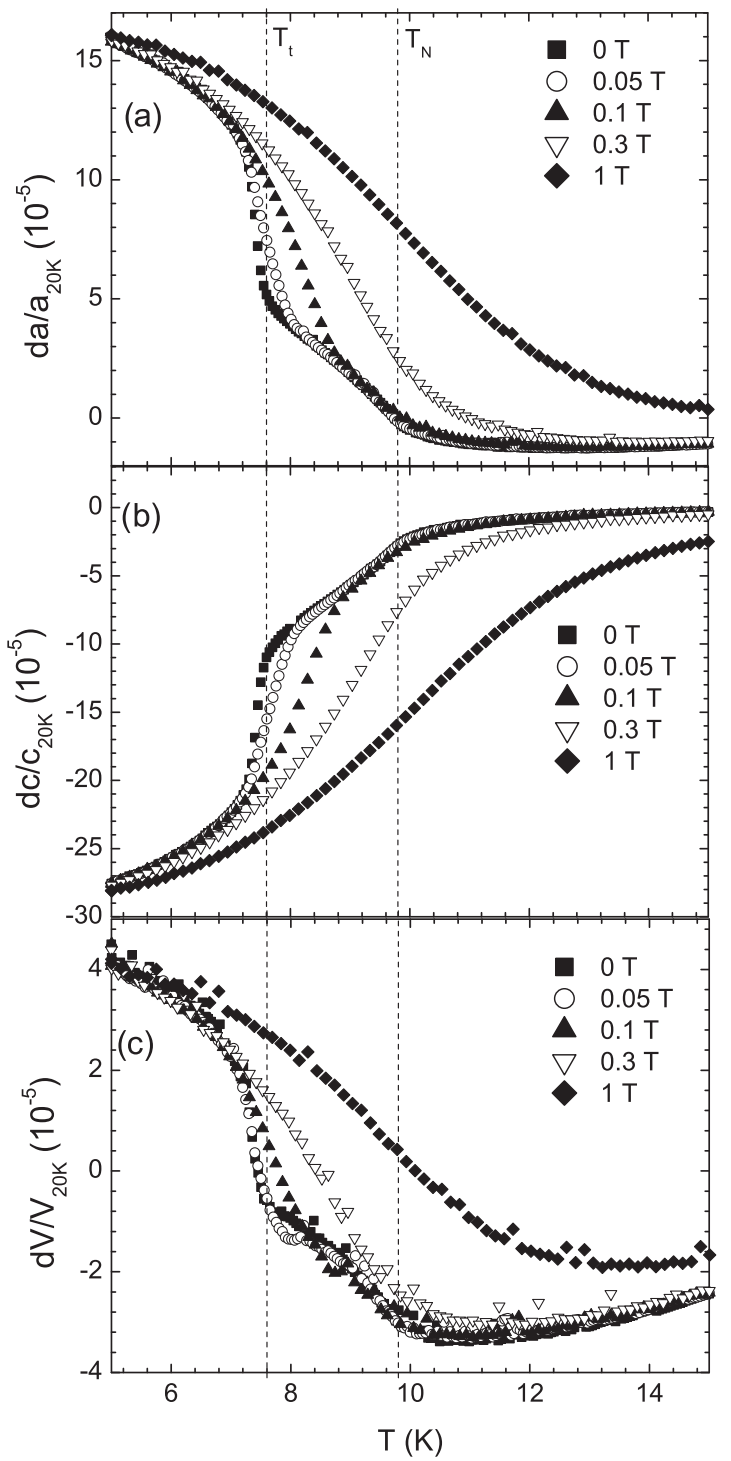

FIG. 7. Relative thermal expansion of NdRhSn in various magnetic fields up to $1 \mathrm{~T}$ applied along the $c$ axis: (a) Measurement along the $a$ axis, (b) measurement along the $c$ axis, and (c) volume evolution as calculated using the $a$ - and $c$-axis data.

\section{E. Elastic properties}

Both magnetic transitions at $T_{N}$ and $T_{t}$ are connected with a shrinking of the unit cell along the $c$ axis and an increase of the distances in the basal plane (Fig. 7). This causes a change of the exchange interaction between the $\mathrm{Nd}^{3+}$ moments and hence a change of the magnetic structure.

Because of the strong magnetocrystalline anisotropy, the external field was applied along the $c$ axis (EMD) only. The increasing field induces a smoothing of the antiferromagneticferromagnetic (F-AF) transition at first. Above some critical value of the field, completely smooth variations of the lattice parameters are observed, accompanied by an increase of the temperature of this transition with increasing field.

The critical field $H_{c}$ of the AF-F transition can be clearly determined from the magnetostriction measurements that have been done at temperatures between 6 and $12 \mathrm{~K}$ (see Fig. 8).

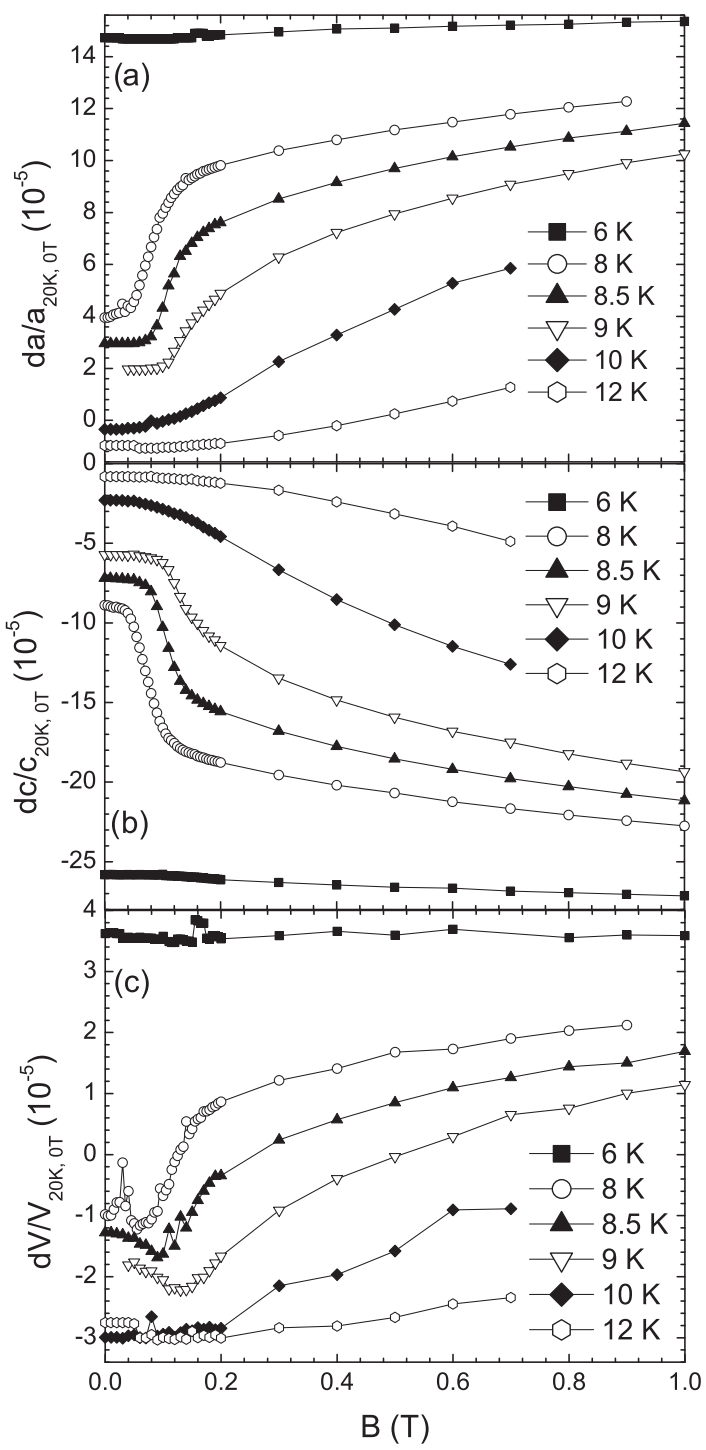

FIG. 8. (a) $a$ - axis and (b) $c$-axis relative magnetostriction of $\mathrm{NdRhSn}$. The reference points are the values measured at $0 \mathrm{~T}$ and $20 \mathrm{~K}$. (c) Calculated relative change of the volume using the $a$ - and $c$-axis data. In all cases, the magnetic field was applied along the $c$ axis. 
The suppression of the antiferromagnetic phase by an external field and the magnetostrictive changes of the lattice parameters are clearly relevant to the results of the thermal-expansion measurements at increasing external field. Because $H_{c}$ is only slightly temperature dependent, the sharp character of the AF-F transition visible in the magnetostrictive changes of the lattice parameters is preserved up to $T_{N}$. The competition of the abrupt expansion of the lattice parameter $a$ and the simultaneous contraction of $c$ [Figs. 8(a) and 8(b)] with increasing field results into a minimum in the volume versus field curve [Fig. 8(c)], just very close to the metamagnetic transition in the magnetization measurements (Fig. 4 in Ref. 12).

The Clausius-Clapeyron relation can be written in the form

$$
\frac{d T}{d P}=V_{M} \frac{\left(\frac{\Delta V}{V}\right)}{\Delta S},
$$

where $V_{M}$ is the molar volume, $\Delta S$ is the entropy change, and $(\Delta V / V)$ is the relative change of the volume during the phase transition. This equation can be used to predict how the temperatures $T_{N}$ and $T_{t}$ shift under applied hydrostatic pressure. In the case of hydrostatic pressure, the relative change of the volume during the magnetic phase transitions can be derived from the thermal-expansion measurements presented in Fig. 7 by

$$
\frac{\Delta V}{V}=2 \frac{\Delta a}{a}+\frac{\Delta c}{c},
$$

where $\Delta a / a$ and $\Delta c / c$ are the relative changes of the lattice parameters. The thermal expansion at temperatures lower than $15 \mathrm{~K}$ approaches zero and can be neglected. To be consistent with the estimations of the magnetic entropy presented in Sec. IV A, we have ascribed all changes of the lattice parameters at $2.5<T<8.4 \mathrm{~K}$ to the phase transition at $T_{t}$ and the changes of the lattice parameters at $8.4<T<12 \mathrm{~K}$ to the phase transition at $T_{N}$. It implies that at paramagnetic-antiferromagnetic phase transition the lattice parameters jump by $\Delta a / a=-4.26 \times 10^{-5}$ and $\Delta c / c=+6.56 \times 10^{-5}$. Similarly, for the antiferromagneticferromagnetic phase transition we obtain $\Delta a / a=-1.37 \times$ $10^{-4}$ and $\Delta c / c=+2.15 \times 10^{-4}$. The entropy changes $\Delta S$ at $T_{N}$ and $T_{t}$ are presented in the section dealing with the specific heat. From the XRD data at room temperature (Table I), a value for the molar volume $V_{M}$ equal to $58.9 \mathrm{~cm}^{3} \mathrm{~mol}^{-1}$ can be derived that is very similar to $V_{M}=59.3 \mathrm{~cm}^{3} \mathrm{~mol}^{-1}$, obtained from the neutron-diffraction data at $14 \mathrm{~K}$. Inserting the derived values for $\Delta V / V, \Delta S$, and $V_{M}$ in Eq. (1), we find $d T_{N} / d p=-0.6 \mathrm{~K} / \mathrm{GPa}$ and $d T_{t} / d p=-1 \mathrm{~K} / \mathrm{GPa}$.

In the case of uniaxial compression of the $\mathrm{NdRhSn}$ single crystal along the $c$ axis, we cannot directly compare the isotropic volume change during the magnetic transitions with the anisotropic uniaxial compression of the sample. Neglecting the shear stress at the edges of the sample, the lattice parameter $c$ will decrease and the lattice parameter $a$ will increase owing to Poisson's ratio $v$. Using only this information, we cannot make any estimation of how $T_{N}$ and $T_{t}$ will behave under the uniaxial compression. However, we will present the experimental findings in the next sections.

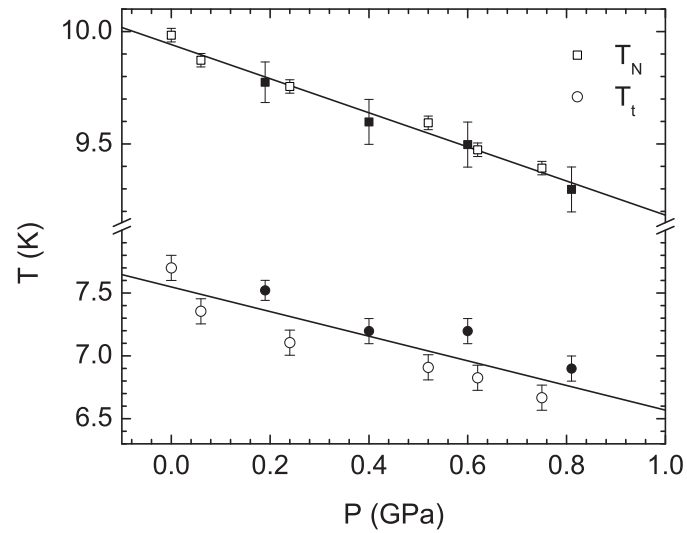

FIG. 9. Evolution of the magnetic-transition temperatures $T_{N}$ and $T_{t}$ of NdRhSn under applied hydrostatic pressure. The filled symbols represent the results from experiment No. 1 and the open symbols represent the results from experiment No. 2. In both cases, the data have been taken at $B=0.01 \mathrm{~T}$ with $B \| c$ axis.

\section{F. Bulk pressure experiments}

The observed huge magnetocrystalline anisotropy, together with the very pronounced anisotropy of the thermal expansion and the magnetostriction of $\mathrm{NdRhSn}$ in a relatively narrow temperature range, have been the main reasons for performing the standard high-hydrostatic-pressure experiments together with the uniaxial-pressure experiments. The first aim was to verify the predicted tendency in the pressure behavior of the magnetic-ordering temperatures $T_{t}$ and $T_{N}$ under hydrostatic compression and to determine the behavior under uniaxial compression. It was found that, upon application of hydrostatic pressure, both $T_{N}$ and $T_{t}$ shift linearly to lower temperatures with $\Delta T_{N} / \Delta p=$ $-0.76(5) \mathrm{K} / \mathrm{GPa}$ and $\Delta T_{t} / \Delta p=-0.9(2) \mathrm{K} / \mathrm{GPa}$ (Fig. 9). Completely different behavior is observed if uniaxial pressure is applied along the $c$ axis. In this case, both transitions shift linearly to higher temperatures with $\Delta T_{N} / \Delta p=+1.2(5) \mathrm{K} / \mathrm{GPa}$ and $\Delta T_{t} / \Delta p=+2.7(4) \mathrm{K} / \mathrm{GPa}$ (Fig. 10).

Upon application of hydrostatic (uniaxial) pressure, the metamagnetic transition shifts to higher (lower) magnetic

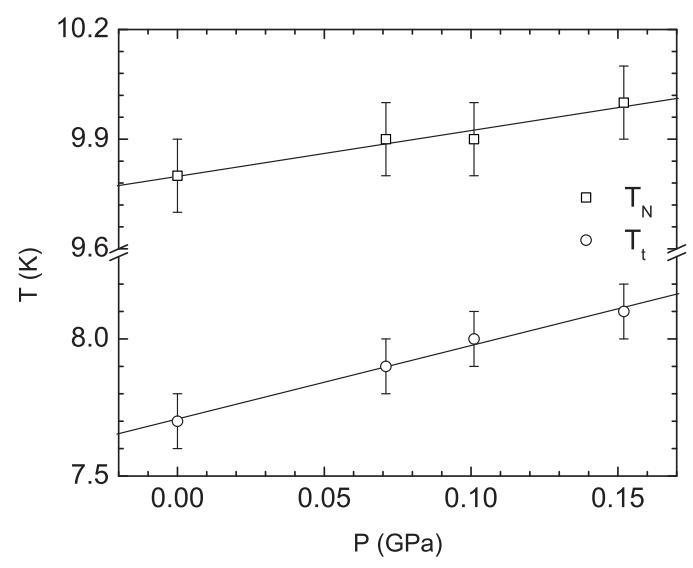

FIG. 10. Evolution of the transition temperatures $T_{N}$ and $T_{t}$ of $\mathrm{NdRhSn}$ at $B=0.01 \mathrm{~T}$ under uniaxial pressure. The data have been taken for the experimental configuration $B\|p\| c$ axis. 


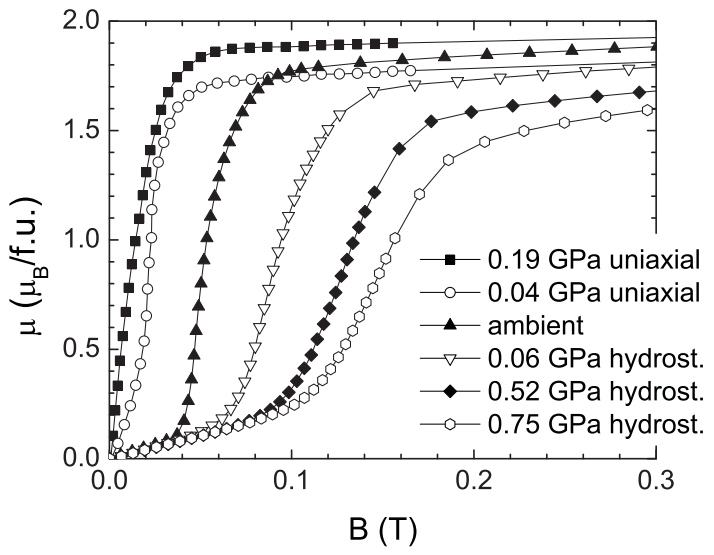

FIG. 11. Comparison of the evolution of the metamagnetic transition in NdRhSn at $T=8.2 \mathrm{~K}$ with the field applied parallel to the $c$ axis under hydrostatic and uniaxial pressure. The data under hydrostatic pressure are from experiment No. 2 and the zero-pressure data have been obtained in the same hydrostatic-pressure experiment in the unclamped hydrostatic-pressure cell.

fields (Fig. 11). It is necessary to add that the magnetization measured at $2 \mathrm{~K}$ (our lowest attainable temperature) remains unchanged upon application of hydrostatic pressure (Fig. 12), which is consistent with the localized nature of the $\mathrm{Nd}$ $4 f$ electrons bearing the $\mathrm{Nd}$ magnetic moment. On the other hand, an extraordinary large and positive effect of uniaxial pressure applied along the $c$ axis was observed in the narrow temperature range of the antiferromagnetic phase (Fig. 12). This effect is fully compatible with the observed decrease of the critical field $H_{c}$ of the metamagnetic transitions above $T_{t}$ in a single crystal under uniaxial pressure.

The NdRhSn intermetallic compound is a natural layered system with the ferromagnetic $\mathrm{Nd}-\mathrm{Rh}$ layers that are separated by nonmagnetic Rh-Sn layers going along the crystal $c$ axis. Based on the review article of Gignoux

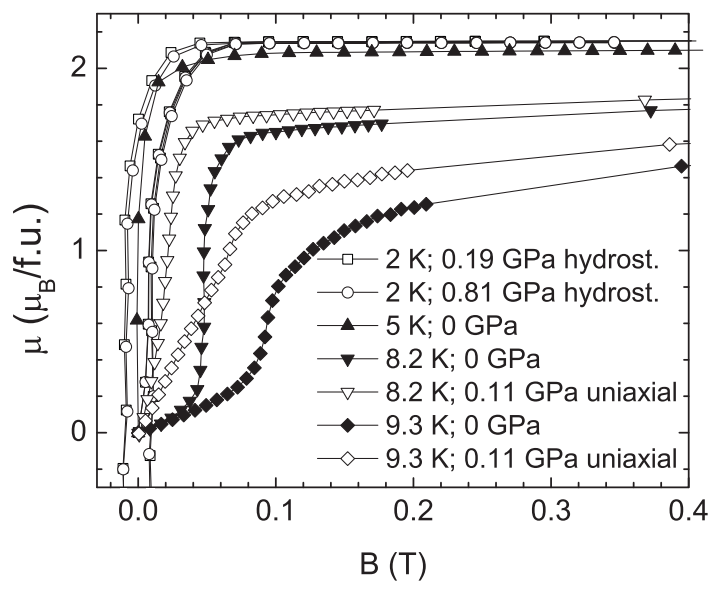

FIG. 12. First-quadrant part of the hysteresis loops of NdRhSn at $2 \mathrm{~K}$ measured at different hydrostatic pressures, together with hysteresis loops at a uniaxial pressure of $0.11 \mathrm{GPa}$ along the $c$ axis at 8.2 and $9.3 \mathrm{~K}$. In all cases, the magnetic field was applied along the $c$ axis. The hydrostatic pressure data were obtained in experiment No. 1. and Schmitt, ${ }^{27}$ we extrapolate that the indirect interaction between the layers in the $\mathrm{NdRhSn}$ is the Rudermann-KittelKasuya-Yoshida (RKKY) interaction. This interaction is long range and oscillatory in dependence on the distance between the layers, i.e. on the crystal $c$-parameter in our case. Hence, taking into consideration the increase of the $c$ parameter in course of the temperature induced transition from ferro- into antiferromagnetic order, we can assume a monotonous decrease of the RKKY interaction and the change of its sign with increasing $c$ parameter in a range of values of the $c$ parameters in view (see the thick line in Fig. 13). The observed incommensurate antiferromagnetic structure has been described by the propagation vector $\left(\begin{array}{lll}0 & 0 & t_{z}\end{array}\right)$, so the interaction between $\mathrm{Nd}$ moments in the Nd-Rh layers remains ferromagnetic. We can present the following simple, phenomenological model that can shed light on at least the pressure behavior of this interaction. In the case of the uniaxial compression of the NdRhSn intermetallics along the $c$ axis, the crystal $c$ parameter decreases and the $a$ parameter increases, with almost zero change in the elementary cell volume. The RKKY interaction increases along the almost unchanged $J(c)$ dependence (arrows "U" in Fig. 13). The uniaxial compression of NdRhSn shifts the antiferromagnetic system closer to the ferromagnetic state. The critical field $H_{c}$ of the metamagnetic transition decreases and the temperature $T_{t}$ increases. The relatively large change in volume of $\mathrm{NdRhSn}$ under hydrostatic pressure has to change the $J(c)$ dependence to be relevant to a system with smaller interatomic distances (arrows " $\mathrm{H}$ " in Fig. 13). The antiferromagnetic interaction between the ferromagnetic layers increases, the critical field $H_{c}$ of the metamagnetic transition increases, and the temperature $T_{t}$ decreases.

To describe properly the transitions from the antiferromagnetic to paramagnetic state, simultaneously taking into account the slight increase of $T_{N}$ under uniaxial pressure and its decrease under hydrostatic pressure, additional assumptions

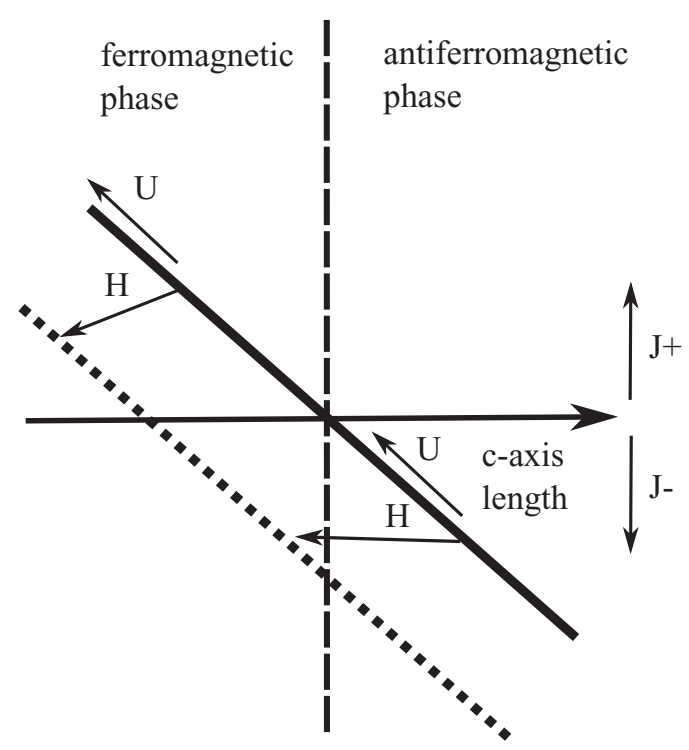

FIG. 13. The sketch of the proposed local RKKY interaction (thick lines) acting along the $c$ axis. The changes of the local interaction according to the hydrostatic $(\mathrm{H})$ and uniaxial $(\mathrm{U})$ pressure are plotted as small arrows. 


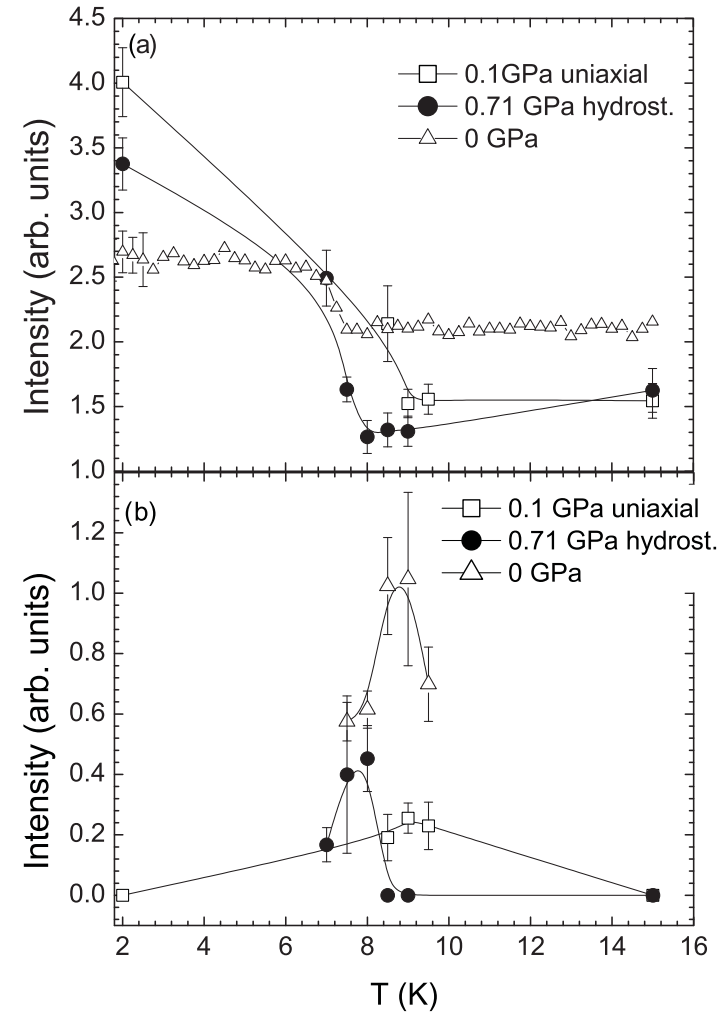

FIG. 14. Temperature evolution of the integrated intensity of (a) the nuclear $\left(\begin{array}{lll}2 & 0 & 0\end{array}\right)$ reflection of $\mathrm{NdRhSn}$ and (b) its magnetic satellite. The lines are guides to the eye.

have to be added. Because these assumptions are not experimentally underlined, we will not present them. A lack of these additional assumptions makes a deeper phenomenological model impossible to establish.

\section{G. Neutron diffraction of NdRhSn under pressure}

It was found that the position of magnetic satellites does not shift in $Q$ space under applied uniaxial or hydrostatic pressure. This implies that neither hydrostatic pressure up to $0.71 \mathrm{GPa}$, nor uniaxial pressure up to $0.1 \mathrm{GPa}$ forces the change of the magnetic structure from the observed one at ambient pressure. The only observed effect is a shift of the maximum intensity of the satellites to lower (higher) temperatures upon application of hydrostatic (uniaxial) pressure (Fig. 14). This trend is in accordance with generally observed trends of the magneticordering temperatures $T_{t}$ and $T_{N}$ under relevant pressures.

Within the experimental error, the intensity and position of the magnetic satellites remain unaffected by the applied magnetic field until the field reaches values equal to the critical field of the metamagnetic transition at comparable hydrostatic (uniaxial) pressure. Above this field, the magnetic satellites abruptly vanish [Fig. 15(b)] and the intensity of the nuclear $\left(\begin{array}{lll}2 & 0 & 0\end{array}\right)$ reflection increases [Fig. 15(a)]. It means that the metamagnetic transition observed in the magnetization is in all cases a spin-flip transition and the magnetic phase at magnetic fields above $0.2 \mathrm{~T}$ ( $B \| c$ axis) is the field-induced ferromagnetic state. This is in agreement with the absence of a metamagnetic transition at high magnetic fields.

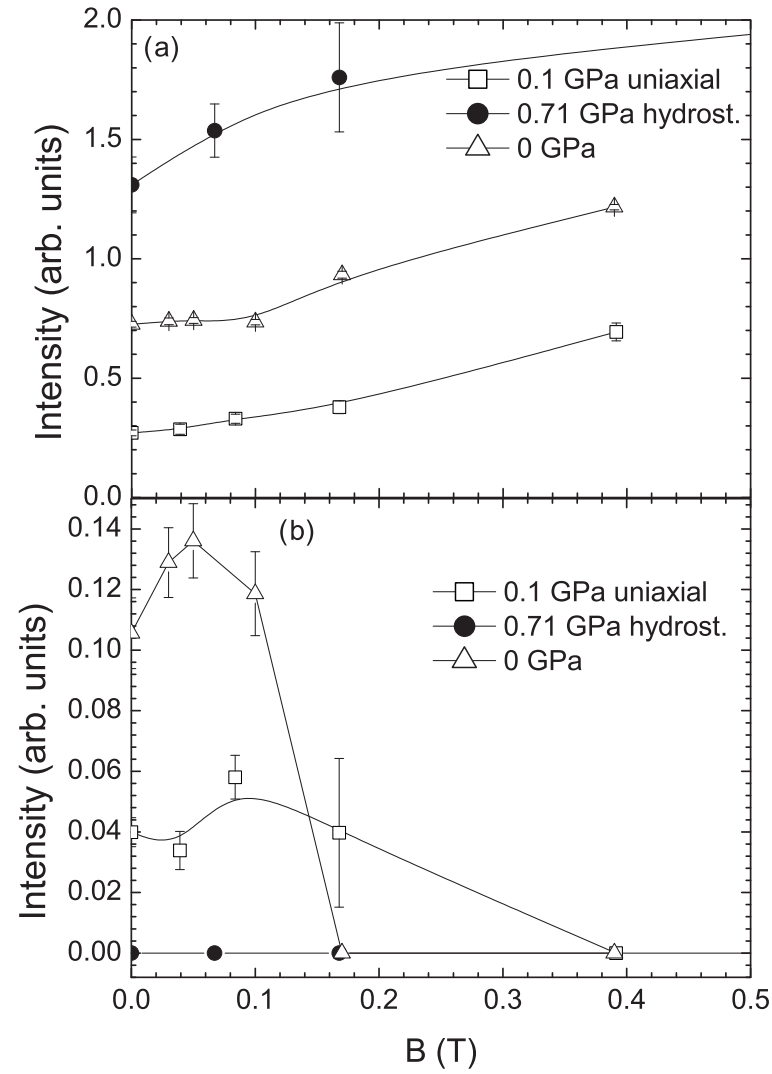

FIG. 15. Magnetic-field evolution at $9 \mathrm{~K}$ of the integrated intensity of (a) the nuclear (2 00 ) reflection and (b) its magnetic satellite. In all cases, the magnetic field was applied along the $c$ axis. The lines are guides to the eye.

\section{CONCLUSIONS}

We have established the existence of two magnetic phase transitions in NdRhSn: a first transition at $T_{N}=9.8(1) \mathrm{K}$ from the paramagnetic state to an antiferromagnetic structure with propagation vector $k=(001 / 11)$, and a second transition at $T_{t}=7.6(1) \mathrm{K}$ to the ferromagnetic state with the magnetic moment aligned along the $c$ axis. In both magnetic states, only the $\mathrm{Nd}^{3+}$ ions carry a magnetic moment. This moment amounts to 2.2(1) $\mu_{B} / \mathrm{Nd}^{3+}$ in the ferromagnetic phase. Both magnetic phases of $\mathrm{NdRhSn}$ exhibit an opposite reaction upon hydrostatic pressure and uniaxial pressure applied along the $c$ axis: Hydrostatic pressure shifts both transition temperatures to lower temperatures and increases the magnetic field needed to destroy the antiferromagnetic state, whereas uniaxial pressure shifts both transitions to higher temperatures and decreases the field needed to destroy the antiferromagnetic phase. The pressure experiments and their good match with the theoretical predictions based on the thermal-expansion data indicate that the two magnetic phase transitions in this material are promoted by strong magnetoelastic interactions that are manifested by an anomalous anisotropic spontaneous magnetostriction, i.e., the change of the $c / a$ ratio at $T_{t}$ and $T_{N}$. This points to a quite complex character of the interatomic, magnetic, and phonon interactions at low temperatures in the compound NdRhSn. 


\section{ACKNOWLEDGMENTS}

This work is a part of the research plan MSM 0021620834 that is financed by the Ministry of Education of the Czech Republic and has been also partly supported by the Czech Science Foundation Grants No. 202/09/1027 and
No. 202/09/P354. Neutron-scattering experiments were supported by the European Commission under the 6th FP through the Key Action: Strengthening the European Research Infrastructures, Contract No. RII3-CT-2003-505925 (NMI 3). *matus.mihalik@helmholtz-berlin.de

${ }^{1}$ A. Szytuła, in Handbook of Magnetic Materials, Vol. 6 (Elsevier, Amsterdam, 1991), Chap. 2, pp. 85-180.

${ }^{2}$ A. E. Dwight, W. C. Harper, and C. W. Kimball, J. Less-Common Met. 30, 1 (1973).

${ }^{3}$ F. Canepa and S. Cirafici, J. Alloys Compd. 232, 71 (1996).

${ }^{4}$ M. Mihalik, V. Sechovský, M. Diviš, S. Gabáni, and M. Mihalik, J. Alloys Compd. 452, 241 (2008).

${ }^{5}$ M. S. Kim, Y. Echizen, K. Umeo, T. Tayana, T. Sakakibara, and T. Takabatake, Physica B 329-333, 524 (2003).

${ }^{6}$ K. Łątka, R. Kmieć, J. Gurgul, M. Rams, A. Pacyna, T. Schmidt, and R. Pöttgen, J. Solid State Chem. 178, 3101 (2005).

${ }^{7}$ M. Mihalik, H. Kitazawa, M. Diviš, and V. Sechovský, J. Alloys Compd. 460, 26 (2008).

${ }^{8}$ C. D. Routsi, J. K. Yakinthos, and H. Gamari-Seale, J. Magn. Magn. Mater. 117, 79 (1992).

${ }^{9}$ K. Łạtka, R. Kmieć, R. Kruk, A. W. Pacyna, M. Rams, T. Schmidt, and R. Pöttgen, Nukleonika 48, S35 (2003).

${ }^{10}$ K. Ła̧tka, R. Kmieć, J. Gurgul, A. W. Pacyna, M. Rams, T. Schmidt, and R. Pöttgen, J. Magn. Magn. Mater. 301, 359 (2006).

${ }^{11}$ M. Mihalik, M. Diviš, J. Kamarád, and V. Sechovský, Physica B 387, 161 (2007).

${ }^{12}$ M. Mihalik, J. Kamarád, and V. Sechovský, J. Magn. Magn. Mater. 316, e415 (2007).

${ }^{13}$ A. M. Mulders, A. Yaouanc, P. Dalmas de Réotier, P. C. M. Gubbens, A. A. Moolenaar, B. Fåk, E. Ressouche, K. Prokeš, A. A. Menovsky, and K. H. J. Buschow, Phys. Rev. B 56, 8752 (1997).
${ }^{14}$ N. R. Wilson, O. A. Petrenko, and L. C. Chapon, Phys. Rev. B 75, 094432 (2007).

${ }^{15}$ D. R. Behrendt, S. Legvold, and F. H. Spedding, Phys. Rev. 108, 1544 (1958).

${ }^{16}$ [http://www.ill.eu/sites/fullprof/].

${ }^{17}$ J. Kamarád, M. Mihalik, V. Sechovský, and Z. Arnold, High Press. Res. 28, 633 (2008).

${ }^{18}$ M. Rotter, H. Müller, E. Gratz, M. Doerr, and M. Loewenhaupt, Rev. Sci. Instrum. 69, 2742 (1998).

${ }^{19}$ J. Kamarád, Z. Machátová, and Z. Arnold, Rev. Sci. Instrum. 75, 5022 (2004).

${ }^{20}$ A. Eiling and J. S. Schilling, J. Phys. F 11, 623 (1981).

${ }^{21}$ M. Mihalik, B. Janoušsová, V. Sechovský, T. Komatsubara, S. Daniš, and J. Vejpravová, Physica B 378-380, 150 (2006).

${ }^{22}$ M. Kolenda, S. Baran, A. Oleś, N. Stüsser, and A. Szytuła, J. Alloys Compd. 269, 25 (1998).

${ }^{23}$ Ł. Gondek, S. Baran, A. Szytuła, D. Kaczorowski, and J. Hernández-Velasco, J. Magn. Magn. Mater. 285, 272 (2005).

${ }^{24}$ L. Keller, A. Dönni, H. Kitazawa, J. Tang, F. Fauth, and M. Zolliker, Physica B 241-243, 660 (1998).

${ }^{25}$ K. H. J. Buschow, in Ferromagnetic Materials, Vol. 1, edited by E. P. Wohlfarth (North-Holland, Amsterdam, 1980), pp. 297-414.

${ }^{26}$ A. Szytuła, B. Penc, and N. Stüsser, J. Magn. Magn. Mater. 265, 94 (2003).

${ }^{27}$ D. Gignoux and D. Schmitt, J. Magn. Magn. Mater. 100, 99 (1991). 